\title{
Dying from takotsubo syndrome at a young age: the crucial role of brain-heart interactions
}

\author{
Francesco Pelliccia ${ }^{1,2}$, Andrea Moretti ${ }^{1}$, Giuseppe Marazzi ${ }^{2}$, Carlo Gaudio ${ }^{1}$ \\ 1Department of Cardiovascular Sciences, Sapienza University, Rome, Italy \\ ${ }^{2}$ IRCCS San Raffaele Pisana, Rome, Italy
}

Adv Interv Cardiol 2018; 14, 3 (53): 221-224

DOI: https://doi.org/10.5114/aic.2018.78323

Takotsubo syndrome (TTS) is characterized by severe left ventricular dysfunction that typically recovers spontaneously within days or weeks [1]. TTS is typically described in association with sudden and severe emotional or physical stressors [2]. Symptoms, clinical signs, and echocardiographic and electrocardiographic findings in TTS patients are suggestive of an acute coronary syndrome [3]. At presentation, patients usually complain of chest pain and dyspnea, but TTS can also present as syncope and pulmonary edema. Cardiac arrest, cardiogenic shock, and serious ventricular arrhythmias occur more rarely in TTS patients. The most frequent finding on the admission electrocardiogram is ST-segment elevation, which most often is present in the precordial leads. Typically, TTS patients manifest modest increases in creatine kinase- $\mathrm{MB}$ and cardiac troponin concentrations as compared to myocardial infarction patients. Of interest, in TTS, there is a disparity between the degree of biomarker elevation and extent of myocardial dysfunction observed on left ventriculography. Diagnostic coronary angiography shows normal coronary arteries or non-obstructive coronary artery disease in the vast majority of patients [4]. Different patterns of LV dysfunction have been reported in TTS, including the classical apical variant, a mid-ventricular variant, a basal or inverted variant and regional variants. The prognosis of TTS was initially thought to be benign. Subsequent series, however, have demonstrated that both acute and long-term mortality are higher than previously recognized. Indeed, mortality reported during the acute phase in hospitalized patients is $\sim 4-5 \%$, a frequency comparable to that of ST-elevation myocardial infarction in the era of primary percutaneous coronary interventions. A recent meta-analysis of clinical correlates of acute mortality in TTS reported that the average in-hospital mortality is $4.5 \%$ [5]. Japanese inves- tigators have recently pointed out that TTS is associated with an elevated in-hospital mortality due to co-existing chronic comorbidities and acute medical illnesses [6]. Of note, major adverse events, including cardiogenic shock, cardiac arrest and mortality, are more frequent in women than in men with TTS.

About $90 \%$ of patients with TTS are post-menopausal females with a similar prevalence across ethnic groups [7]. Conversely, the occurrence of TTS in the young is very uncommon. Recently, Urbinati et al. performed a systematic review of published case reports in patients younger than 18 years [8]. Overall, they found a total of 37 cases of TTS in young patients with a median age of 8 years, of whom $46 \%$ were boys and $54 \%$ were girls. Presentation of TTS in these patients was dramatic, with the majority of patients presenting to hospital with loss of consciousness or symptoms related to heart failure. A classical form of TTS, with apical left ventricular wall motion abnormalities, was seen in 20 cases, while atypical forms of TTS were observed in 17 cases. At admission, ST segment abnormalities were present in 21 cases, troponin and natriuretic peptides levels were usually abnormal, and median left ventricular ejection fraction was $30 \%$. When coronary angiography was performed, epicardial coronary arteries were normal. As regards predisposing factors, it is worth noting that $59 \%$ of patients had underlying neurological or psychiatric disorders, i.e. intracerebral arterial or venous malformations and brain cancers. Neurological triggers were also common and were often related to acute hemorrhages, brain trauma or hypoxia. Other trigger factors were emotional or physical stress, including surgery, drug administration or withdrawal, and infections. The outcome was usually benign in most young patients, with progressive recovery of left ventricular function to normal in a few days, though 
2 patients died ( 1 of them due to cardiac arrest the day after presentation).

In this issue of the journal [9], Zalewska-Adamiec et al. describes the case of a 15-year-old girl with history of ventricular arrhythmias previously diagnosed as having mitral and tricuspid valves prolapse, who experienced loss of consciousness while performing a secondary school test. Notably, the young student's medical history included long lasting stressful conditions prior to the acute event. The girl experienced sudden cardiac arrest and pulseless electrical activity. Resuscitation maneuvers were immediately undertaken by the school personnel and then continued by the Emergency Medical Service. The patient had electrocardiographic evidence of ventricular fibrillation and was defibrillated twice. On hospital admission, she had sinus tachycardia, ST-segment elevation in the precordial leads, and an increase in troponin levels. Invasive assessment showed normal coronary arteries, the typical left ventricular angiographic ballooning pattern, and an ejection fraction of $25 \%$. Despite the progressive improvement in left ventricular function in the subsequent days, the patient remained unconscious and brain death was diagnosed.

This case report is of utmost importance for several reasons. First, it demonstrates that TTS can occur at any age despite most cases being seen in post-menopausal, elderly women. Second, it clearly shows that the condition can be occasionally fatal, though the majority of patients have a benign outcome even when there is severe cardiac function impairment at initial presentation. Third, it contributes to our understanding of the pathophysiology of TTS, emphasizing the crucial role of brain-heart interactions. The reasons why severe emotional or physical stress triggers TTS in certain people but not others

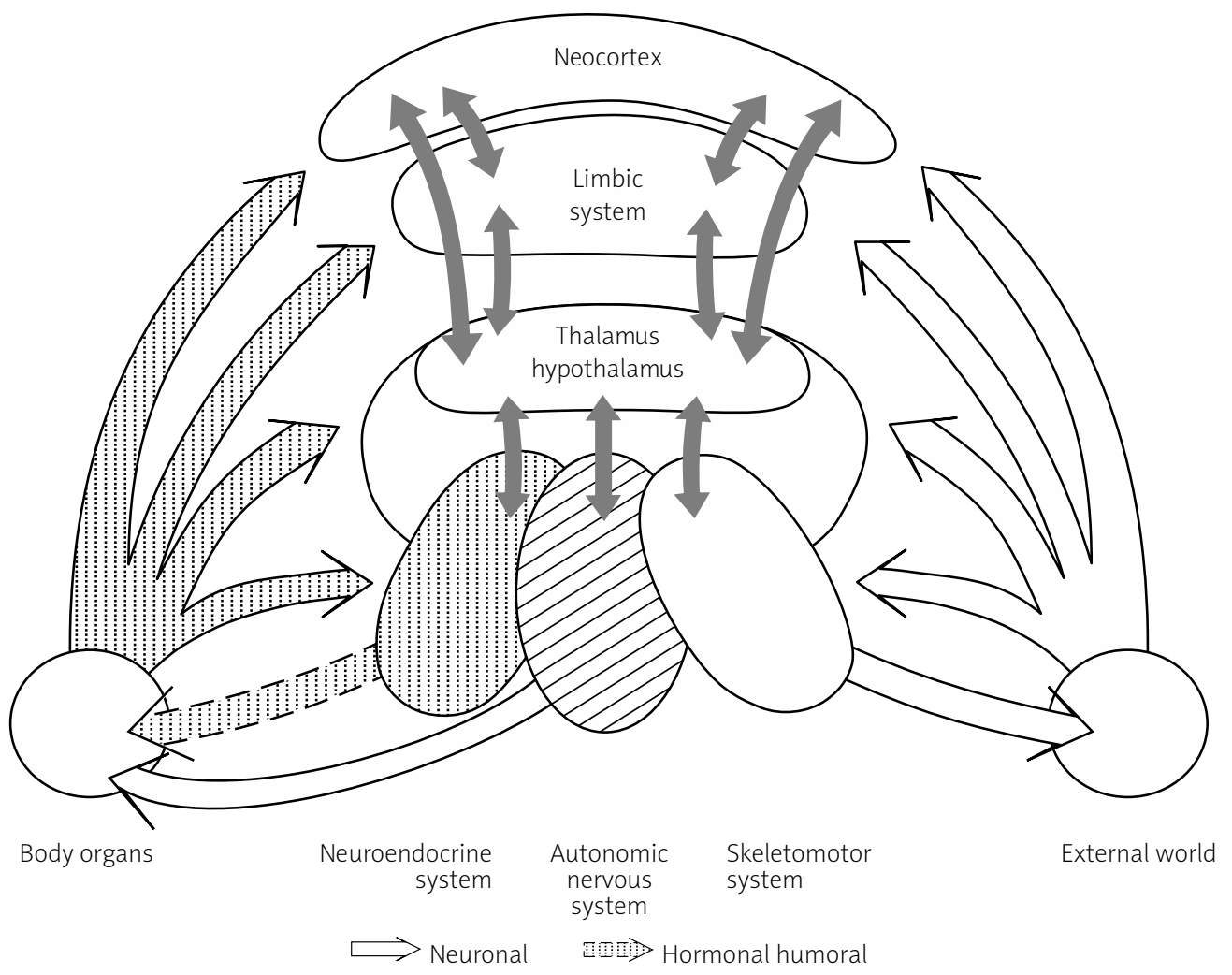

Figure 1. Central and autonomic nervous system interplay. Right, somatic nervous system (motor system and sensory systems) and environment. Left, autonomic nervous system, neuroendocrine system and body organs. In the middle, spinal cord, brain stem, hypothalamus' limbic system and neocortex. The afferent feedback from the body is neuronal, hormonal and humoral (physicochemical; e.g., glucose concentration, osmolality) and of other types (e.g., body temperature). Solid line arrows, neuronal; dashed line arrow, hormonal; dotted, neuroendocrine system, hormonal and humoral feedback. Limbic system is anatomically descriptive and a collective term denoting brain structures common to all mammals that include hippocampus, dentate gyrus with archicortex, cingulate gyrus, septal nuclei and amygdala. These forebrain structures are functionally heterogeneous and not a unitary system (as the term "limbic system" may imply). They are involved in the generation of emotional and motivational aspects of behavior). Note the reciprocal communication between the hypothalamus, limbic system and neocortex (symbolized by the shaded arrows), indicating that the centers of the cerebral hemispheres have a powerful influence on all autonomic regulations 
is still a matter of speculation but strongly suggests the existence of predisposing factors/mechanisms.

In a sizeable proportion of patients several stressors have been identified to precede the onset of TTS [10]. Emotional or psychological stress due to the unexpected death of a relative or a friend, suppressed terror, or the occurrence of natural disasters or strenuous physical stress usually precede the onset of TS [11]. About one in five patients, however, do not report any form of stress preceding the onset of the condition. Stress is a physiological response that mediates the action of a stressor on its target organ. The anatomical structures that mediate the stress response are found in both the central nervous system and the peripheral tissues. Acute emotional stressors have been shown to induce brain activation, increasing bioavailability of cortisol, epinephrine and norepinephrine. The fundamental anatomical structures involved in the stress response are the neocortex, the limbic system, the reticular formation, the brain stem and the spinal cord [12]. Psychosocial stressors are either real or imagined environmental events that elicit the stress response. Specifically, psychosocial stimuli do not act directly, but excite nonspecific arousal and cognitive appraisal mechanisms. Efferent impulses project back to the emotional arousal limbic system, especially the hippocampus, where the cognitive interpretation occurs and visceral effector mechanisms are triggered (Figure 1) [13]. If the appraisal of the stimulus is ultimately one of threat, challenge, or aversion, then emotional arousal will most likely take place. Following the complex neocortical and limbic integrations that occur in the interpretation of a stimulus as "threatening", the neural stress response first occurs through activation of brain stem noradrenergic neurons and sympathetic adrenomedullary circuits, leading to secretion of catecholamine. The principal site for brain synthesis of norepinephrine is the locus coeruleus, which receives afferents from the hypothalamus, the cingulate gyrus and the amygdala, is activated by stress, and responds by increasing norepinephrine secretion. This in turn alters cognitive function through the prefrontal cortex, increases sympathetic discharge and inhibits parasympathetic tone through the brainstem, and activates the hypothalamic pituitary adrenal axis. The secretion of adrenal medullary catecholamines constitutes the hormonal output of the neuroendocrine stress-response axis.

Available evidence clearly shows that catecholamines are a key part of the pathogenesis of TTS, as they can trigger an acute episode of myocardial ischemia, leading to acute left ventricular systolic dysfunction and cardiac stunning [1]. The crucial role of myocardial ischemia in TTS has emerged thanks to the evidence that most cases of TTS occur in patients with risk factors for coronary disease and/or co-morbidities, including neurologic, psychiatric, pulmonary, kidney, liver and connective tis- sue disease [10], which are associated with endothelial dysfunction and might therefore constitute a previously unrecognized predisposing factor for TTS [2]. Indeed, endothelial dysfunction could explain the propensity to epicardial and/or microvascular coronary artery spasm of the disease [14]. This pathologic state of the endothelium, characterized by an imbalance between vasoconstricting and vasodilating factors, may represent an important link between stress and myocardial dysfunction in TTS [1]. In this respect, coronary microvascular dysfunction seems to play a major role. Small coronary arteries and arterioles receive autonomic innervation and are the principal determinants of coronary vascular resistance. In normal subjects the overall response to sympathetic activation is vasodilatation mainly through activation of coronary $\beta_{2}$-adrenoceptors. By contrast, in patients with endothelial dysfunction, increased cardiac sympathetic activity can induce $\alpha$-adrenoceptor mediated coronary microvascular constriction powerful enough to provoke myocardial ischemia [15]. In summary, there is now agreement that the increased concentration of catecholami-

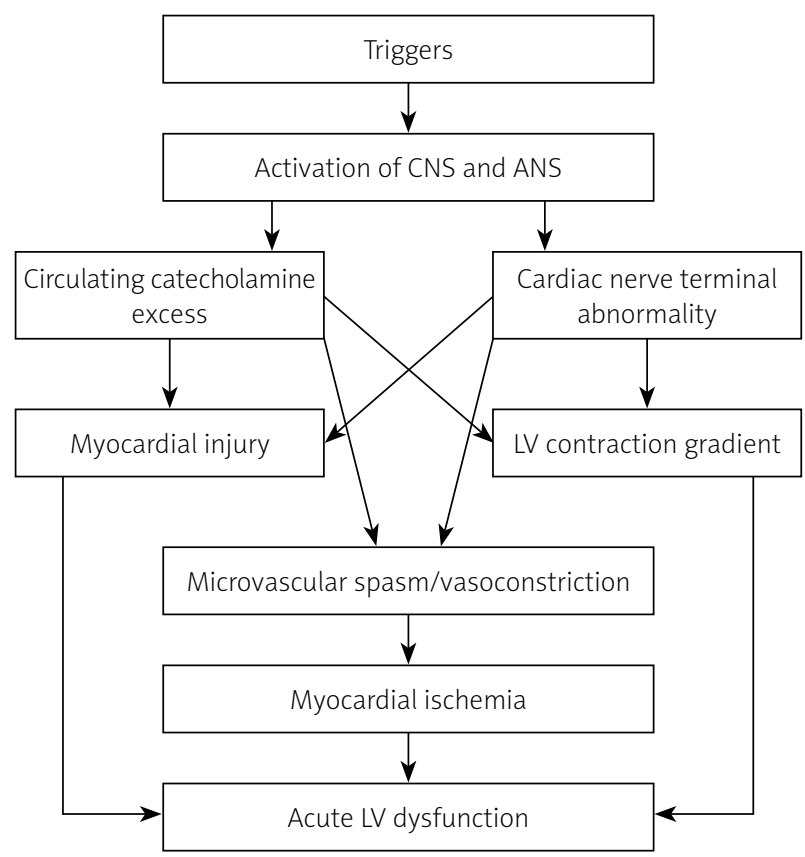

Figure 2. Key pathogenetic aspects in Takotsubo syndrome. The picture depicts the interplay among triggers, pathogenetic factors, mechanisms of cardiac injury, and clinical consequences. Current evidence indicates that in the acute phase of TTS there is an increased concentration of catecholamines that might induce direct myocardial injury and coronary spasm, mostly at the microvascular level, together with an increased cardiac workload that contributes to an acute situation of 'supply-demand mismatch' followed by post-ischemic stunning

ANS - autonomic nervous system, CNS - central nervous system. 
ne occurring in the acute phase of TTS induces direct myocardial injury and coronary vasoconstriction, mostly at the microvascular level, together with an increased cardiac workload, which contribute to an acute situation of 'supply-demand mismatch' followed by post-ischemic stunning. The clinical counterpart is the typical, reversible LV ballooning followed by complete functional recovery in most cases (Figure 2) [1].

\section{Conflict of interest}

The authors declare no conflict of interest.

\section{References}

1. Pelliccia F, Kaski JC, Crea F, et al. Pathophysiology of Takotsubo syndrome. Circulation 2017; 135: 2426-41.

2. Pelliccia F, Greco C, Vitale C, et al. Takotsubo syndrome (stress cardiomyopathy): an intriguing clinical condition in search of its identity. Am J Med 2014; 127: 699-704.

3. Ghadri JR, Wittstein IS, Prasad A, et al. International expert consensus document on Takotsubo syndrome (part I): clinical characteristics, diagnostic criteria, and pathophysiology. Eur Heart $J$ 2018; 39: 2032-204.

4. Ghadri JR, Wittstein IS, Prasad A, et al. International expert consensus document on Takotsubo syndrome (part II): diagnostic workup, outcome, and management. Eur Heart J 2018; 39: 2047-62.

5. Singh K, Carson K, Shah R, et al. Meta-analysis of clinical correlates of acute mortality in Takotsubo cardiomyopathy. Am J Cardiol 2014; 113: 1420-8.

6. Isogai T, Yasunaga $\mathrm{H}$, Matsui $\mathrm{H}$, et al. Out-of-hospital versus in-hospital Takotsubo cardiomyopathy: analysis of 3719 patients in the diagnosis procedure combination database in Japan. Int J Cardiol 2014; 176: 413-7.

7. Templin C, Ghadri JR, Diekmann J, et al. Clinical features and outcomes of Takotsubo (stress) cardiomyopathy. N Engl J Med 2015; 373: 929-38.

8. Urbinati A, Pellicori P, Guerra F, et al. Takotsubo syndrome in the paediatric population: a case report and a systematic review. J Cardiovasc Med 2017; 18: 262-7.

9. Zalewska-Adamiec M, Bachórzewska-Gajewska H, Kralisz P, et al. Sudden cardiac arrest in the course of the Takotsubo syndrome in fifteen-year-old girl. Kardiol Interw 2018; 14: 318-9.

10. Pelliccia F, Parodi G, Greco C, et al. Comorbidities frequency in Takotsubo syndrome: an international collaborative systematic review including 1109 patients. Am J Med 2015; 128: 654.e11-9.

11. Y-Hassan S. Acute cardiac sympathetic disruption in the pathogenesis of the takotsubo syndrome: a systematic review of the literature to date. Cardiovasc Revasc Med 2014; 159: 35-42.

12. Crossman AR, Neary D. Neuroanatomy. $2^{\text {nd }}$ edn. Churchill Livingston, London 2000.

13. Janig W. The Integrative Action of the Autonomic Nervous System. Cambridge University Press, Cambridge, UK 2006.

14. Vitale C, Rosano GM, Kaski JC. Role of coronary microvascular dysfunction in Takotsubo cardiomyopathy. Circ J 2016; 80: 299-305.

15. Crea F, Camici PG, Bairey Merz CN. Coronary microvascular dysfunction: an update. Eur Heart J 2014; 35: 1101-11. 